\title{
CALIDAD DEL AGUA DEL ESTERO EL SAUCE, VALPARAÍSO, CHILE CENTRAL
}

\author{
Water quality in the El Sauce estuary, Valparaíso, Central Chile
}

\begin{abstract}
Cecilia Andrea RIVERA CASTRO ${ }^{1 *}$, Jaime Andrés LETELIER PINO², Betzabé ACEVEDO PIZARRO ${ }^{3}$, Tamara del Pilar TOBAR CORREA ${ }^{1}$, Catalina Loreto TORRES LEPE ${ }^{1}$ Anita María CATALDO FIGUEROA ${ }^{1}$, Anny RUDOLPH GEISSE ${ }^{4}$ y Miguel Ángel RIVERA CASTRO ${ }^{5}$
\end{abstract}

${ }^{1}$ Departamento de Química, Facultad de Ciencias Naturales y Exactas, Universidad de Playa Ancha, Av. Carvallo 270, Playa Ancha, Valparaíso, Chile

${ }^{2}$ Departamento de Oceanografía y Medio Ambiente (DOMA), Instituto de Fomento Pesquero (IFOP), calle Blanco 839, Valparaíso, Chile

${ }^{3}$ Departamento de Ciencias Químicas, Facultad de Ciencias Exactas, Universidad Andres Bello, calle Quillota 980, CP 2520000, Viña del Mar, Chile

${ }^{4}$ Facultad de Ciencias, Universidad Católica de la Santísima Concepción, Av. Alonso de Ribera 2850, Concepción, Chile

${ }^{5}$ Departamento de Ciencias Sociales Aplicadas, Universidad Estatal de Feira de Santana, CEP 44036-900, Feira de Santana, Bahía, Brasil; Programa de Postgrado en Administración, Universidad de Salvador, CEP 40301155, Salvador de Bahía, Brasil

*Autora para correspondencia: cecilia.rivera@upla.cl

(Recibido: noviembre 2018; aceptado: enero 2019)

Palabras clave: aguas superficiales, contaminación, eutrofización, Laguna Verde

\section{RESUMEN}

Este trabajo tuvo por objetivo principal evaluar la calidad del agua del estero El Sauce en toda su extensión y sus afluentes. La cuenca del estero El Sauce se ubica en la localidad de Laguna Verde, Valparaíso, Chile Central. La toma de muestras se realizó en la época estival de 2013 y 2015, en 11 estaciones ubicadas a lo largo de la cuenca, cinco de ellas distribuidas desde su origen hasta su desembocadura en el mar y seis ubicadas antes del ingreso en sus afluentes. Se identificaron fuentes puntuales y no puntuales descargadas en su curso. Destaca por su volumen, en la zona de origen del estero, la descarga directa de una planta de tratamiento de aguas servidas, y en su zona media el ingreso de percolados de un relleno sanitario municipal. En tanto, su desembocadura se ve afectada por fuentes no puntuales de aguas domésticas en la localidad de Laguna Verde. Los resultados muestran que el estero es un curso de agua somero, que en gran parte de su extensión presenta calidad Clase 4 (mala) debido al contenido de materia orgánica, nutrientes, cloruros y contaminación fecal, por lo cual incumple con la normativa ambiental para cualquier uso. Se observan falta de planes de manejo y de fiscalización en la utilización de este importante recurso, cuyo uso se ha convertido en un riesgo para la comunidad. Ésta utiliza el agua del estero para el riego de una agricultura de subsistencia y recreación con contacto directo en su desembocadura.

Key words: surface water, pollution, eutrophication, Laguna Verde 


\begin{abstract}
The main objective of this work was to evaluate the water quality of the El Sauce estuary and its tributaries. The El Sauce estuary basin is located in the town of Laguna Verde, Valparaíso, Central Chile. Sampling took place in the summer season of 2013 and 2015, in 11 stations located along the basin, five of them distributed from its origin to its mouth in the sea and six located before entering its tributaries. Point and non-point sources downloaded in its course were identified. The direct discharge of water from a sewage treatment plant in the area of origin of the estuary, and in its middle zone the percolation of a municipal landfill, stand out for their volume. Its mouth is affected by non-point sources of domestic waters in the town of Laguna Verde. The results show that the estuary is a shallow water course, which quality Class 4 (poor) in most of its extension presents due to the content of organic matter, nutrients, chlorides, and fecal contamination, not complying with environmental regulations for any use. There is a lack of management and control plans in the use of this important resource. It has become a risk to the community, who use the water of the stream both to irrigate subsistence agriculture and for recreation with a direct contact at its mouth.
\end{abstract}

\section{INTRODUCCIÓN}

El deterioro en la calidad de las aguas superficiales de fácil acceso para la población, que la utiliza para servicios básicos y actividades de esparcimiento, se ha convertido en motivo de preocupación a nivel mundial (Figueroa et al. 2007, Azpilcueta et al. 2017), debido a que los cuerpos de agua continentales muestran importantes alteraciones en su ciclo hidrológico (Tóth 2000, Wolanski et al. 2004). Del mismo modo, la utilización de sus hábitats ha producido una importante pérdida de su valor ecosistémico (de Groot et al. 2007). Esta situación ha generado que cuerpos de agua dulce, como ríos y esteros, que son fundamentales para la supervivencia de su población aledaña, se convierten en fuentes de riesgos para la salud (Smith et al. 1999, OMS 2005). Este deterioro se debe a factores como crecimiento de la población, expansión de la actividad industrial y agrícola, cambios ambientales asociados al cambio climático y principalmente, a la falta de información científica básica que permita implementar normas secundarias de calidad y establecer medidas de manejo adecuadas para su recuperación y sostenibilidad.

El presente estudio se desarrolló en el estero El Sauce (Fig. 1), un cuerpo de agua dulce superficial ubicado en la región de Valparaíso (Chile Central). Este estero se ubica en la zona sur de la localidad de Placilla, y se origina en la zona de confluencia entre el estero Las Cenizas (que tiene como principal tributario a la laguna El Peral) y el estero La Luz (que recibe aguas de Laguna La Luz y Lago Peñuelas). Esta cuenca forma parte de la zona de transición del corredor biológico de la Reserva de la Biósfera de La Campana-Peñuelas (Moreira y Salazar 2014).

Hasta 2005, el principal uso de esta cuenca fue proveer a la comunidad de Placilla y Laguna Verde de agua para consumo humano y el desarrollo de una agricultura básica de subsistencia (Aranda 2013, Zúñiga 2015). Sin embargo, la calidad del agua de este importante recurso ha experimentado un paulatino deterioro (Millanir 2003, Silob 2012), aunque sigue utilizándose para el riego en el sector rural y con uso recreativo (zona de balneario) en su desembocadura (DGA 2005, Tobar y Torres 2014). Referencias de los habitantes del poblado indican que este estero constaba de una zona ribereña que podría ser clasificada como de clase humedal o laguna costera permanente (Figueroa et al. 2009), la cual servía de estación de parada de aves migratorias, hábitat de poblaciones de peces tales como pejerrey y gambusia, entre otros, y pequeños mamíferos como coipos, los cuales en la actualidad han desaparecido (Zunino et al. 2009).

Así, el presente estudio tuvo como objetivo evaluar la calidad del agua en el estero El Sauce en toda su extensión y aportar información básica para un mejor manejo de este recurso. Para ello se determinaron las fuentes contaminantes en su curso, y se seleccionaron y evaluaron parámetros físicos, químicos y microbiológicos. Los resultados fueron comparados con los criterios de calidad establecidos en la legislación chilena (Norma Chilena Oficial 1333/Of 78 modificada 1987), correspondiente a la "Norma de Calidad de Agua para diferentes usos, como es el de riego, conservación de vida acuática y recreación"; además, con la categorización propuesta en la Guía CONAMA para el establecimiento 
de "Normas Secundarias de calidad ambiental para aguas continentales superficiales" (CONAMA 2004), mediante 17 parámetros físicos y químicos entre los que destacan la demanda bioquímica de oxígeno $\left(\mathrm{DBO}_{5}\right)$ como medida de la materia orgánica, y cloruros y nutrientes para identificar posibles fuentes de contaminación doméstica o industrial, entre otros. Esta normativa establece los siguientes tipos de calidad de agua: excepcional (Clase 0), muy buena (Clase 1), buena (Clase 2), regular (Clase 3 ) y mala (Clase 4). Según esta clasificación, las aguas aptas para su captación y potabilización corresponden a las clases 0 a 3 (excepcional regular), mientras que las aguas que excedan los límites establecidos para la Clase 3 corresponden a la clasificación de mala calidad, es decir, no adecuadas para la conservación de las comunidades acuáticas y su utilización requiere de tratamiento. Además, se compararon los resultados obtenidos para la cuenca con normas internacionales.

\section{MATERIAL Y MÉTODOS}

\section{Área de estudio}

El estero El Sauce, que pertenece a La Cuenca homónima, es un cauce superficial que posee una extensión de $6.4 \mathrm{~km}$ con superficie estimada de
$204 \mathrm{~km}^{2}$. Se ubica en la localidad rural de Laguna Verde $\left(33^{\circ} 05^{\prime} \mathrm{S}-71^{\circ} 35^{\prime} \mathrm{O}\right)$ en la sección sur de la Comuna de Valparaíso (Fig. 1). El estero se origina en la bifurcación de los esteros de régimen pluvial Las Cenizas y La Luz, para desembocar en la zona costera conocida como Playa Grande de Laguna Verde, con una cuenca aportante de $5708016 \mathrm{~m}^{3}$. En gran parte de su extensión el uso de sus aguas es principalmente agrícola y doméstico (DGA 2016), aunque en la actualidad su desembocadura se utiliza con fines recreativos (Tobar y Torres 2014).

Su principal aporte hídrico natural proviene de un pequeño humedal, la laguna El Peral (5.01 ha), a través del estero Las Cenizas, el cual recorre $7.70 \mathrm{~km}$ en la localidad de Placilla y se transforma finalmente en el estero El Sauce en la localidad de Laguna Verde (Fig. 1). La zona presenta un marcado régimen pluvial de abril a septiembre, con precipitación promedio de $677.2 \mathrm{~mm}$ (DGA 2005). El estero recibe además aportes del lago Peñuelas (1433 ha) y la laguna La Luz (77.9 ha). Se recarga, además, durante el periodo estival de aguas subterráneas del pueblo de Laguna Verde (PLADECO 2006).

\section{Selección de las estaciones de muestreo}

En el cuadro I se indican las coordenadas geográficas y la distancia a la desembocadura de las

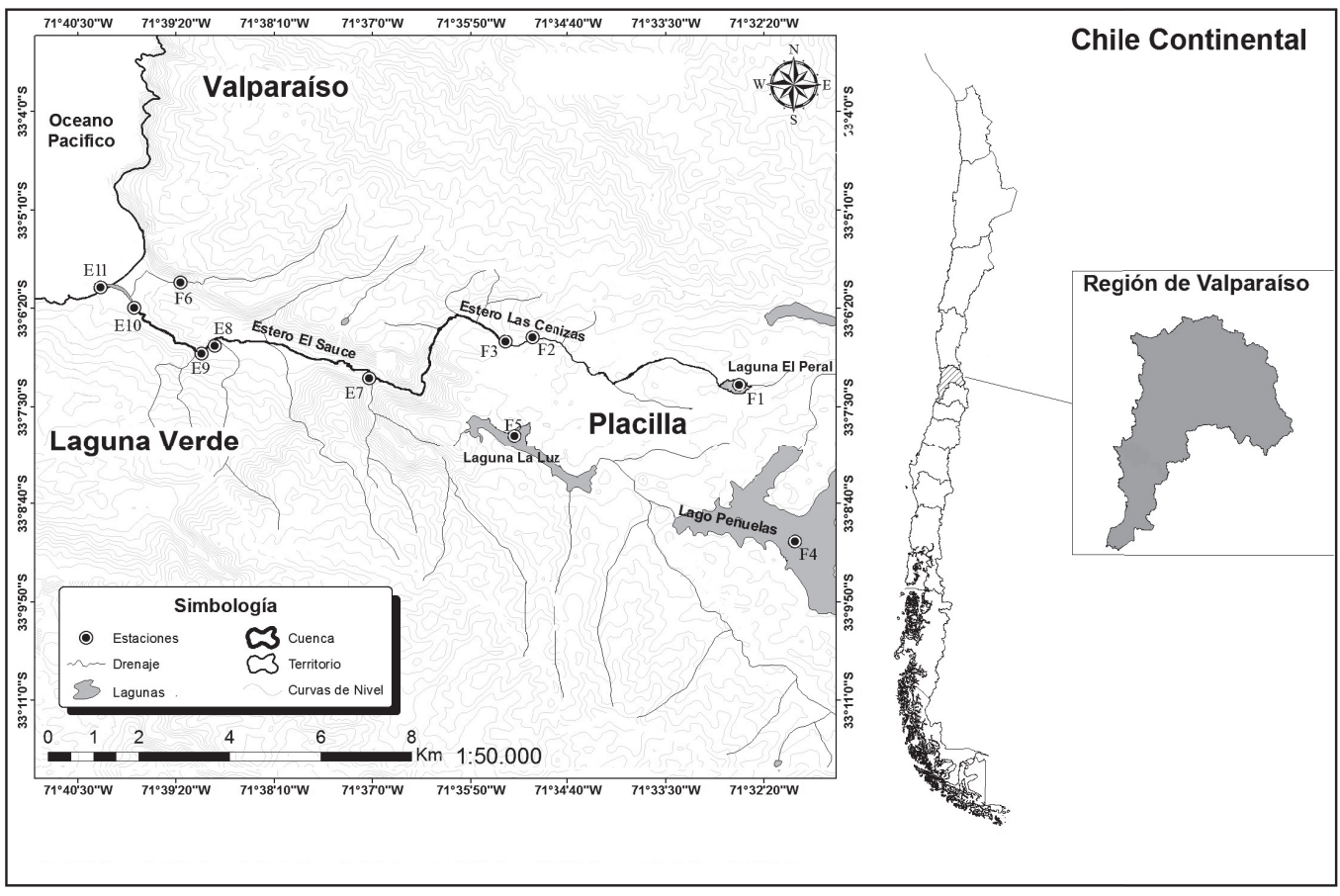

Fig. 1. Localización geográfica de la cuenca estudiada y de las estaciones de muestreo ubicadas entre Laguna Verde y Placilla, Valparaíso, Chile 
CUADRO I. NOMBRE Y CARACTERÍSTICAS GEOGRÁFICAS DE LAS ESTACIONES EN ESTUDIO

\begin{tabular}{|c|c|c|c|c|c|c|c|}
\hline \multirow[t]{2}{*}{ Estación } & \multirow[t]{2}{*}{ Cuerpo de agua } & \multirow[t]{2}{*}{ Localidad } & \multirow[t]{2}{*}{ Detalle ubicación } & \multicolumn{2}{|c|}{ Coordenadas } & \multirow{2}{*}{$\begin{array}{l}\text { Altitud } \\
\text { (m) }\end{array}$} & \multirow{2}{*}{$\begin{array}{l}\text { Distancia } \\
\text { al mar (m) }\end{array}$} \\
\hline & & & & (S) & $(\mathrm{O})$ & & \\
\hline F1 & Laguna El Peral & Placilla & Zona norte & $33^{\circ} 7^{\prime} 13.24^{\prime \prime}$ & $71^{\circ} 32^{\prime} 43.55^{\prime \prime}$ & 373.0 & 11855 \\
\hline $\mathrm{F} 2$ & Estero Las Cenizas & Placilla & $\begin{array}{l}\text { Antes de la descarga de } \\
\text { RILES Esval }\end{array}$ & $33^{\circ} 6^{\prime} 48.52^{\prime \prime}$ & $71^{\circ} 34^{\prime} 39.63^{\prime \prime}$ & 325.8 & 8883 \\
\hline F3 & Descarga de RILES & Placilla & $\begin{array}{l}\text { RILES de la planta de } \\
\text { tratamiento Esval }\end{array}$ & $33^{\circ} 6^{\prime} 44.50^{\prime \prime}$ & $71^{\circ} 34^{\prime} 47.00^{\prime \prime}$ & 323.8 & 8681 \\
\hline F4 & Lago Peñuelas & Placilla & Ribera norte frente cancha & $33^{\circ} 8^{\prime} 53.26^{\prime \prime}$ & $71^{\circ} 33^{\prime} 21.05^{\prime \prime}$ & 345.5 & 12258 \\
\hline F5 & Laguna La Luz & Placilla & Frente compuertas & $33^{\circ} 7^{\prime} 52.82^{\prime \prime}$ & $71^{\circ} 35^{\prime} 13.92^{\prime \prime}$ & 333.2 & 8583 \\
\hline F6 & Agua de escorrentía & Laguna Verde & Cascada Cuesta ruta F98-G & $33^{\circ} 6^{\prime} 1.51^{\prime \prime}$ & 71³9'16.51”' & 69.0 & 1609 \\
\hline E7 & Estero El Sauce & Laguna Verde & Fundo El Sauce, origen & $33^{\circ} 7^{\prime} 9.92 ”$ & $71^{\circ} 37^{\prime} 5.40^{\prime \prime}$ & 33.9 & 5482 \\
\hline E8 & Estero El Sauce & Laguna Verde & Fundo El Sauce & $33^{\circ} 6^{\prime} 46.72^{\prime \prime}$ & $71^{\circ} 38^{\prime} 52.41^{\prime \prime}$ & 7.1 & 2602 \\
\hline E9 & Estero El Sauce & Laguna Verde & Puente Los Azules & $33^{\circ} 6^{\prime} 51.34^{\prime \prime}$ & $71^{\circ} 39^{\prime} 1.03^{\prime \prime}$ & 5.7 & 2484 \\
\hline E10 & Estero El Sauce & Laguna Verde & Baden & $33^{\circ} 6^{\prime} 19.35^{\prime \prime}$ & 71'39'48.98', & 12.1 & 892 \\
\hline E11 & Estero El Sauce & Laguna Verde & $\begin{array}{l}\text { Desembocadura Playa } \\
\text { Grande }\end{array}$ & $33^{\circ} 6^{\prime} 4.67^{\prime \prime}$ & $71^{\circ} 40^{\prime} 10.75^{\prime \prime}$ & 6.0 & 127 \\
\hline
\end{tabular}

estaciones de muestreo en el estero El Sauce (Fig. 1). Todas las estaciones de muestreo fueron georreferenciadas mediante sistemas de posicionamiento global (GPS) (Garmin etrex, Venture HC, Huso 19). En tanto, la figura 2 muestra imágenes del estero Las Cenizas y el estero El Sauce a lo largo de su extensión.
La toma de muestras en terreno fue realizada en la época estival (18-19 diciembre de 2013 y 15-16 de enero de 2015).

Se seleccionó un total de 11 estaciones de muestreo, seis de ellas ubicadas antes del ingreso de sus afluentes, identificadas como:
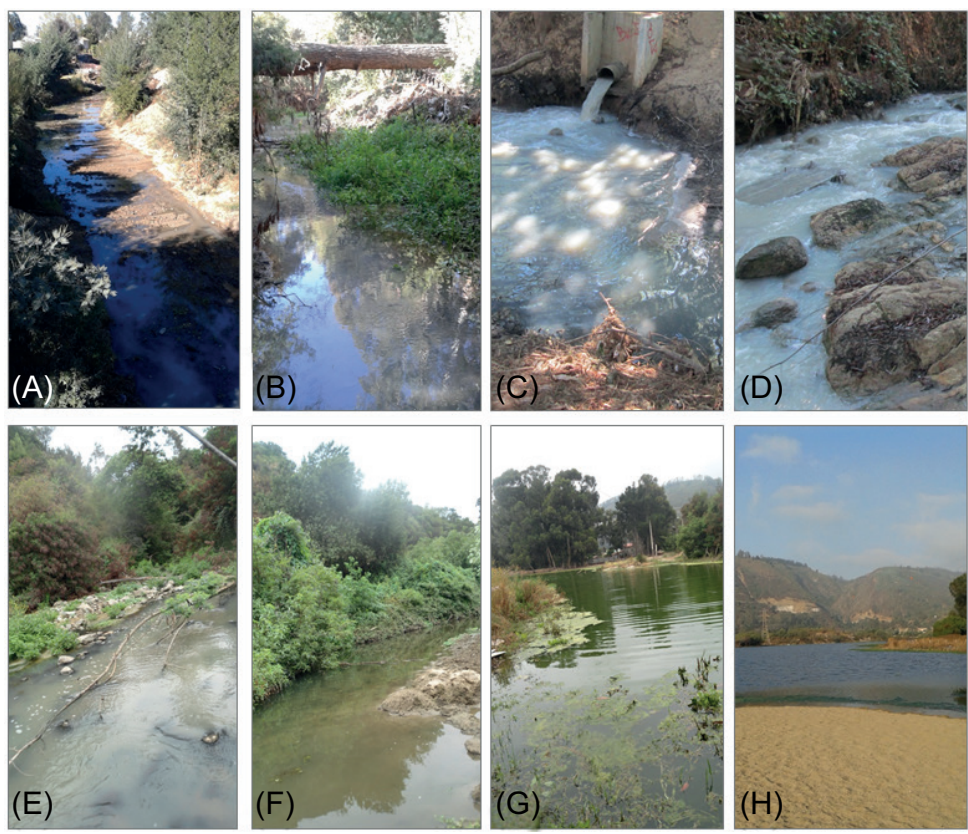

Fig. 2. Imágenes de las estaciones en estudio. (a, b) Estero Las Cenizas antes de la descarga (F2); (c, d) estero Las Cenizas después de la descarga de aguas servidas (F3); (e) estero El Sauce desde su origen en el fundo El Sauce (E7); (f, g) zona centro del estero (E9 y E10), y (h) su desembocadura (E11). 
- F1: Laguna El Peral, fuente puntual y principal aporte natural del estero Las Cenizas, el que aguas abajo cambia de nombre a estero El Sauce

- F2: Estero Las Ceniza, ubicada después de haber atravesado la localidad de Placilla y todas sus fuentes no puntuales presentes en ese trayecto.

- F3: Fuente puntual de la descarga de aguas servidas proveniente de la planta de tratamiento (PTAS) Esval-Placilla, la cual genera un aumento del $70 \%$ de su cauce.

- F4 y F5: Lago Peñuelas y laguna La Luz, humedales artificiales que abastecen al estero de forma subterránea y superficialmente en invierno.

- F6: fuente puntual de aguas de escorrentía superficial permanente (ruta F98-G) en Laguna Verde, que bajan a través de la microcuenca Los Molles y se incorporan al estero en su zona media.

Las otras cinco estaciones fueron ubicadas a lo largo del estero El Sauce (E7-E11) desde su origen (E7) hasta su desembocadura en la Playa Grande de Laguna Verde (E11). De las 11 estaciones monitoreadas F1, F4 y F5, fueron medidas sólo en 2015.

\section{Parámetros físicos, químicos y microbiológicos}

La calidad del agua se evaluó utilizando 17 indicadores físicos, químicos y microbiológicos elegidos según las características ambientales del recurso y su uso (de La Lanza 2000). En todos los puntos muestreados se recopiló información in situ de profundidad (escandallo), transparencia (disco de Secchi), temperatura ambiente y superficie del agua (SM-2550B), pH (SM-4500-H B), conductividad eléctrica (SM-2510-AB) y concentración de oxígeno disuelto (OD) (SM-4500-O G, equipo multiparamétrico Water Quality Meter 8603, Veto y Cía.). Las muestras de agua fueron recolectadas por triplicado $(\mathrm{n}=3)$, siguiendo los protocolos estipulados en la Norma Chilena Oficial 411 (MOP 1996). Las muestras fueron trasladadas para su análisis al Laboratorio de Investigación Ambiental de la Facultad de Ciencias Naturales y Exactas, Universidad de Playa Ancha (UPLA). La metodología utilizada se basó en los métodos estándar (Standard Methods, SM) de la APHA (2005) y los parámetros evaluados fueron: alcalinidad (SM-2320 B), color (SM-2120 C), cloruros $\left(\mathrm{Cl}^{-}\right)(\mathrm{SM}-4500 \mathrm{Cl}-\mathrm{B})$, dureza total (Dur T) (SM-1240 C), sólidos sedimentables (SS) (SM-2540 F), contenido de coliformes totales y fecales (SM9221-B, $\mathrm{n}=5$, diluciones $\left.10^{-2}\right)$, materia orgánica como $\mathrm{DBO}_{5}(\mathrm{SM}-4500-\mathrm{O} \mathrm{G})$, nitrato $\left(\mathrm{NO}_{3}{ }^{-}\right)(\mathrm{SM}-$ $\left.4500 \mathrm{NO}_{3}{ }^{-} \mathrm{B}\right)$, nitritos $\left(\mathrm{NO}_{2}{ }^{-}\right)\left(\mathrm{SM}-4500 \mathrm{NO}_{2}{ }^{-} \mathrm{B}\right) \mathrm{y}$ fosfatos $\left(\mathrm{PO}_{4}^{-3}\right)$ mediante equipo Hanna HI-95717.
Los resultados fueron comparados con los límites máximos de una compilación de diferentes normas nacionales e internacionales indicadas en el cuadro II. El análisis de la información de nutrientes y calidad de agua se realizó mediante distribución t de Student y análisis multivariable de componentes principales (ACP). Se utilizó una matriz de correlación para asociar las variables ambientales con los sitios y periodos de estudio (Jongman et al. 1987). Los análisis fueron realizados en el programa PAST v. 3.21 (Hammer et al. 2001).

\section{RESULTADOS Y DISCUSIÓN}

La región de Valparaíso (Chile Central) presenta un importante deterioro de la calidad de sus aguas en las zonas rurales y costeras, debido principalmente al desarrollo acelerado de la industria, que genera grandes cantidades de residuos contaminantes. Además, el incremento de la población y su concentración en las ciudades superan la capacidad de la infraestructura de tratamiento de aguas residuales domésticas, las cuales están siendo evacuadas en los cursos del agua, sobrepasando la capacidad de asimilación del ambiente (UNESCO 2017). Esto, sumado a la escasa información disponible sobre las características físicas y químicas de los cuerpos de agua dulce y su calidad ecológica (Pavez et al. 2018), ha generado la necesidad de asegurar la calidad de los recursos hídricos como posibles fuentes de abastecimiento a la población, lo que ha evidenciado las malas condiciones de sus aguas. Un ejemplo de ello es la localidad rural de Laguna Verde, la cual posee una agricultura reciente y de baja escala; presenta, además, un crecimiento exponencial en los últimos cinco años en el sector inmobiliario y turístico (Zúñiga 2015), lo que ha generado la necesidad de utilizar las aguas superficiales, generando una problemática ambiental y de salud de su población, debido a su mal estado.

Los resultados obtenidos de los afluentes (Cuadro III) muestran que, de acuerdo con la normativa ambiental, sus aguas corresponden a la categoría de regular calidad (Clase 3) en su origen, correspondiente a la laguna El Peral (F1). Su cauce permanente y superficial abastece al estero Las Cenizas, el cual es somero, con agua dulce acuícola de tipo blanda, abastecido principalmente por agua de lluvia con una conductividad promedio de $114 \mu \mathrm{S} / \mathrm{cm}$; presenta alto contenido de OD, alta transparencia y baja concentración de materia orgánica, cloruros y nitrógeno. Sin embargo, sus valores sobrepasan los límites máximos 
CUADRO II. PARÁMETROS FÍSICOS, QUÍMICOS Y MICROBIOLÓGICOS ESTIMADOS PARAAGUAS SUPERFICIALES. SE INDICAN LOS LÍMITES MÁXIMOS Y NORMAS DE REFERENCIAS UTILIZADAS EN SU ANÁLISIS

\begin{tabular}{lccc}
\hline Parámetros & Unidad & $\begin{array}{c}\text { Límite máximo } \\
\text { permitido }\end{array}$ & $\begin{array}{c}\text { Referencias } \\
\text { normas }\end{array}$ \\
\hline Transparencia & $\mathrm{m}$ & 1.2 & 1 \\
Temperatura agua & $\mathrm{C}$ & $<30$ & 1 \\
$\mathrm{pH}$ & $\mathrm{mg} / \mathrm{L} \mathrm{CaCO}$ & $6.5-9.0$ & 1,2 \\
Alcalinidad & $\mathrm{Pt}-\mathrm{Co}$ & $<20$ & 1 \\
Color & $\mathrm{pS} / \mathrm{cm}$ & $<750$ & 1,3 \\
Conductividad & $\mathrm{mg} / \mathrm{L}$ & 200 & 1 \\
Cloruros & $\mathrm{mg} / \mathrm{L} \mathrm{CaCO}$ & $<100$ & 1 \\
Dureza total & $\mathrm{mL} / \mathrm{L}$ & 1.0 & 2 \\
Sólidos sedimentables & $\mathrm{NMP} \mathrm{en} 100 \mathrm{~mL}$ & 1000 & 4 \\
Coliformes totales & $\mathrm{NMP} \mathrm{en} 100 \mathrm{~mL}$ & 1000 & 1 \\
Coliformes fecales & $\mathrm{mg} / \mathrm{L}$ & $>5$ & 1 \\
Oxígeno disuelto & $\mathrm{mg} / \mathrm{L}$ & $<20$ & 1 \\
Demanda bioquímica de oxígeno 5 & $\mathrm{mg} / \mathrm{L}$ & $<5$ & 3 \\
Nitratos & $\mathrm{mg} / \mathrm{L}$ & $<0.05$ & 2 \\
Nitritos & $\mathrm{mg} / \mathrm{L}$ & $<1.0$ & 2 \\
Fosfatos & & & 2 \\
\hline
\end{tabular}

${ }^{1}$ Norma Chilena Oficial 1333 (MOP 1978), ${ }^{2}$ de la Lanza (2002), ${ }^{3}$ CONAMA (2004),

${ }^{4}$ Luna et al. (2004)

NMP: número más probable

CUADRO III. PARÁMETROS FÍSICOS Y QUÍMICOS MEDIDOS EN LOS AFLUENTES QUE INGRESAN AL ESTERO EL SAUCE, VERANO DE 2013 Y $2015(\mathrm{n}=3)$

\begin{tabular}{|c|c|c|c|c|c|c|c|c|c|c|c|}
\hline \multirow{3}{*}{ Parámetros } & \multirow{3}{*}{$\begin{array}{c}\text { Límite } \\
\text { máximo } \\
\text { permitido }\end{array}$} & \multicolumn{10}{|c|}{ Estaciones } \\
\hline & & \multicolumn{5}{|c|}{2013} & \multicolumn{5}{|c|}{2015} \\
\hline & & E7 & E8 & E9 & E10 & E11 & E7 & E8 & E9 & E10 & E11 \\
\hline Profundidad $\pm 0.01(\mathrm{~m})$ & & 0.12 & 0.45 & 1.15 & 0.61 & 1.08 & 0.10 & 0.08 & 0.24 & 0.25 & 0.45 \\
\hline Transparencia $\pm 0.01(\mathrm{~m})$ & 1.2 & 0.12 & 0.45 & 0.19 & 0.61 & 0.11 & 0.10 & 0.08 & 0.24 & 0.2 & 0.12 \\
\hline Temperatura ambiental $\pm 0.01\left({ }^{\circ} \mathrm{C}\right)$ & & 16.9 & 18.3 & 18.1 & 19.2 & 21.4 & 14.7 & 22.3 & 24.8 & 20.3 & 18.9 \\
\hline Temperatura agua $\pm 0.01\left({ }^{\circ} \mathrm{C}\right)$ & $<30$ & 16.0 & 19.0 & 20.6 & 18.5 & 21.3 & 22.3 & 21.3 & 21.3 & 22.1 & 24.8 \\
\hline $\mathrm{pH} \pm 0.01$ & $6.5-9.0$ & 8.2 & 7.5 & 10.2 & 7.6 & 10.4 & 6.7 & 7.3 & 7.3 & 7.4 & 10.2 \\
\hline Alcalinidad $\pm 0.03\left(\mathrm{mg} / \mathrm{L} \mathrm{CaCO}_{3}\right)$ & $>20$ & 550 & 500 & 350 & 550 & 350 & 240 & 240 & 180 & 180 & 180 \\
\hline Color $\pm 1.0(\mathrm{Pt}-\mathrm{Co})$ & $<100$ & 550 & 432 & 332 & 415 & 350 & 1572 & 78 & 68 & 75 & 225 \\
\hline Conductividad $\pm 0.01(\mathrm{pS} / \mathrm{cm})$ & $<750$ & 1212 & 978 & 1361 & 1081 & 1331 & 1070 & 1017 & 1022 & 1520 & 7071 \\
\hline Cloruros $\pm 0.01(\mathrm{mg} / \mathrm{L})$ & 200 & 355 & 213 & 709 & 142 & 709 & 269 & 199 & 156 & 326 & 2822 \\
\hline Dureza total $\pm 0.01(\mathrm{mg} / \mathrm{L} \mathrm{CaCO} 3)$ & 100 & 160 & 340 & 360 & 240 & 200 & 196 & 196 & 200 & 288 & 704 \\
\hline Sólidos sedimentables $\pm 0.01(\mathrm{~mL} / \mathrm{L})$ & 1.0 & $<1.0$ & $<1.0$ & $<1.0$ & $<1.0$ & $<1.0$ & $<0.1$ & $<0.1$ & $<0.1$ & 0.4 & 1.0 \\
\hline
\end{tabular}

permisibles (LMP) en los parámetros de coliformes totales $(>3000 \mathrm{NMP} / 100 \mathrm{~mL})$ y fecales $(>2400$ $\mathrm{NMP} / 100 \mathrm{~mL}$ ), temperatura y $\mathrm{pH}$ (enero de 2015).

Aguas abajo, en el tramo correspondiente al estero Las Cenizas (F2-F3), en ambos monitoreos se observó que el cauce presenta aguas de mala calidad (Clase 4) debido al aporte de fuentes domésticas no puntuales a lo largo de su curso (caudal $61.5 \mathrm{~m}^{3} / \mathrm{h}$ ), con alto contenido de materia orgánica (50 veces sobre el LMP), alta concentración de coliformes totales (ocho veces sobre la LMP) y fosfatos. Esta situación empeoró en la estación F3 después de atravesar la PTAS con un aumento importante de su caudal $\left(257.5 \mathrm{~m}^{3} / \mathrm{h}\right)$. El contenido de materia orgánica superó los $1400 \mathrm{mg} / \mathrm{L}$, correspondientes a 70 los LMP, además de un aumento en la concentración de fosfato 
(> $6.0 \mathrm{mg} / \mathrm{L}$ ) y coloración (diciembre de 2013) por sobre la norma de emisión.

Los altos valores de $\mathrm{DBO}_{5}$, que superaron los $1000 \mathrm{mg} / \mathrm{L}$ en diciembre 2013 y $99.9 \mathrm{mg} / \mathrm{L}$ en enero de 2015, son característicos de fuentes industriales $(\mathrm{LMP}=20 \mathrm{mg} / \mathrm{L})$. La degradación de altos contenidos de materia orgánica es compleja, ya que desencadena el agotamiento de la concentración de OD y mecanismos como la desnitrificación y sulfatoreducción, pudiendo generarse compuestos tóxicos como amonio y sulfuros de hidrógeno (Schindler 2006, Hargrave et al. 2008).

De acuerdo con la Superintendencia de Servicios Sanitarios (SISS 2013) los altos valores de $\mathrm{DBO}_{5}$ en F2 se debieron en dicha época al vertido ilegal de la planta de Pescadores Group, que fue multada después de su fiscalización. Sin embargo, estos valores (en la estación F3), deberían haber disminuido y no aumentado a $1400 \mathrm{mg} / \mathrm{L}$ por efecto de la dilución que alcanza a más del $76 \%$ del caudal, evidenciándose que la contaminación puntual de la PTAS de Placilla se sigue produciendo, incumpliendo la norma de emisión del Decreto Supremo (DS) 90 (MSGPSEGPRES 2000). Por otra parte, los altos valores de fosfatos (seis veces superiores a los LMP) pueden generar alteraciones en los procesos de las redes tróficas (Chapman y Kimtsach 1996, APHA 2005).

$\mathrm{Al}$ evaluar los resultados de las estaciones en Lago Peñuelas (F4) y Laguna la Luz (F5), ambos ecosistemas se encontrarían en la categorización de aguas de mala calidad. Cabe destacar que sólo en los días de lluvia se transforman en fuentes puntuales del estero en estudio. La evaluación mostró que son humedales de aguas tipo dulce, blanda a moderadamente dura, con baja concentración de cloruros, nitrógeno y materia orgánica, y conductividad $<255 \mu \mathrm{S} / \mathrm{cm}$. Su profundidad fue superior a $0.8 \mathrm{~m}$ en la orilla y estación de muestreo y una cota máxima de $10 \mathrm{~m}$ en la zona centro (Navarro 2013) para F4 y de $23 \mathrm{~m}$ para F5 (PLADECO 2006). La mala calidad se debería a que sus aguas presentaron altos valores en color (sobre $349 \mathrm{Pt}-\mathrm{Co}$, [3.5 y 3.7 veces sobre la norma]), con una pérdida de la transparencia superior al $60 \%$, alta concentración de fosfatos, altos valores de coliformes totales, baja concentración de OD en superficie y altos valores de SS, categorizando a ambos humedales en estado eutrófico.

La estación F6 recibe aguas que recorren gran parte de la zona poblada, incluyendo la escuela rural de la localidad, para desembocar en el estero El Sauce. Los resultados muestran que no son aguas naturales, sino subsalinas (rango $>1500 \mathrm{y}<5000 \mu \mathrm{S} /$ $\mathrm{cm}$ ) (Montes y Martino 1987), con alta conductividad
( $>4010 \mu \mathrm{S} / \mathrm{cm}$ ), alta concentración de cloruros (> $\left.2056 \mathrm{mg} / \mathrm{L} \mathrm{Cl}^{-}\right), 10.3$ veces por sobre los LMP de la Norma Chilena Oficial 1333 (MOP 1978) y 5.1 veces sobre los LMP del DS 90 (MSGP-SEGPRES 2000). Este valor concuerda con residuos industriales líquidos (RIL) de percolados de tipo industrial (León et al. 2015). Además, se registraron altos valores de materia orgánica ( $>104 \mathrm{mg} / \mathrm{L}$ en 2013), nitratos $(59.2$ y $11.8 \mathrm{mg} / \mathrm{L})$, nitritos (>0.17 mg/L) y fosfatos $(>6.4 \mathrm{mg} / \mathrm{L})$, datos concordantes con lo informado por Silob (2012), por lo cual su clasificación correspondería a aguas de mala calidad (Clase 4) en ambos años de estudio.

El relleno sanitario Los Molles se ubica en la zona alta de la cuenca estudiada. Este sector funcionó hasta 2013 como vertedero municipal. Actualmente tiene una capacidad instalada para recibir $1500 \mathrm{t}$ de residuos domésticos por día (Cataldo 2015). Tobar y Torres (2014) e Inostroza y Ruiz (2015) indican que estos percolados no sólo contaminan al estero El Sauce, sino que además al acuífero de la zona del pueblo, de donde sus habitantes extraen el $90 \%$ del agua, la cual utilizan con y sin tratamiento para consumo humano. En este estudio se evaluaron más de 30 pozos del sector, observándose que sus aguas subterráneas son subsalinas de muy mal olor, con alto contenido de nutrientes, cloruros y presencia de coliformes fecales. Encuestas realizadas a sus dueños indicaron que su principal uso es el riego, pero se observó que familias con adultos mayores, que llevan viviendo más de 30 años en la localidad, la consumen sin tratamiento, lo que implica un alto riesgo para su salud.

Al evaluar la calidad del agua del estero El Sauce (Cuadro IV), los resultados muestran que las aguas de este ecosistema presentaron en ambos monitoreos una mala calidad a lo largo de todo su cauce. Esto se debería a que el $76.1 \%$ de sus aguas son de origen industrial, esto es, provenientes de fuentes difusas que ingresan en la localidad de Placilla, sumadas a las aguas de la planta de aguas servidas Esval (F3), ubicada aguas arriba del estero a una distancia de 3.65 $\mathrm{km}$ (Tobar y Torres 2014, Cataldo 2015) y a las aguas de percolados que drenan desde el relleno sanitario Los Molles en la zona norte de Laguna Verde (F6). En cuanto a su profundidad, el estero es somero en gran parte de su cauce, siendo la zona de la desembocadura la más profunda (Fig. 2); presenta baja transparencia, aguas de color grisáceo en su origen y verde oscuro en su desembocadura, con una media de $225 \mathrm{Pt}-\mathrm{Co}$ y valores de $\mathrm{pH}$ alcalinos en la mayoría de su cauce, llegando a pH 10.4 en su desembocadura. En tanto, la alcalinidad presentó valores de $456 \pm 108 \mathrm{mg} / \mathrm{L}$ a 
CUADRO IV. PARÁMETROS FÍSICOS Y QUÍMICOS DE LAS MUESTRAS DE AGUA MEDIDAS EN EL ESTERO EL SAUCE, VERANO DE 2013 Y $2015(\mathrm{n}=3)$

\begin{tabular}{|c|c|c|c|c|c|c|c|c|c|c|}
\hline \multirow{2}{*}{ Parámetros } & \multirow{2}{*}{$\begin{array}{l}\text { Límite } \\
\text { máximo }\end{array}$} & \multicolumn{3}{|c|}{2013} & \multicolumn{6}{|c|}{2015} \\
\hline & & $\mathrm{F} 2$ & F3 & F6 & $\mathrm{F} 1$ & $\mathrm{~F} 2$ & F3 & F4 & F5 & F6 \\
\hline Profundidad $\pm 0.01(\mathrm{~m})$ & & 0.30 & 0.30 & $<0.1$ & 0.70 & 0.18 & 0.15 & 0.85 & 0.80 & $<0.1$ \\
\hline Transparencia $\pm 0.01(\mathrm{~m})$ & 1.2 & 0.30 & 0.28 & $<0.1$ & 0.63 & 0.18 & 0.13 & 0.32 & 0.80 & $<0.1$ \\
\hline Temperatura ambiental $\pm 0.01\left({ }^{\circ} \mathrm{C}\right)$ & & 21.1 & 19.3 & 21.6 & 16.4 & 15.0 & 15.0 & 24.2 & 19.3 & 18.1 \\
\hline Temperatura agua $\pm 0.01\left({ }^{\circ} \mathrm{C}\right)$ & $<30.0$ & 20.3 & 16.8 & 17.0 & 21.9 & 16.6 & 21.4 & 22.0 & 21.0 & 16.9 \\
\hline $\mathrm{pH} \pm 0.01$ & $6.5-9.0$ & 8.0 & 7.2 & 8.0 & 9.0 & 7.2 & 6.7 & 9.3 & 8.4 & 7.5 \\
\hline Alcalinidad $\pm 0.03\left(\mathrm{mg} / \mathrm{L} \mathrm{CaCO}_{3}\right)$ & $>20.0$ & 220 & 800 & 550 & 340 & 300 & 280 & 140 & 140 & 280 \\
\hline Color $\pm 1.0(\mathrm{Pt}-\mathrm{Co})$ & $<100$ & 139 & 448 & 50 & 59 & 126 & 1063 & 349 & 365 & 191 \\
\hline Conductividad $\pm 0.01(\mathrm{pS} / \mathrm{cm})$ & $<750$ & 623 & 988 & 4010 & 114 & 579 & 1040 & 221 & 255 & 5190 \\
\hline Cloruros $\pm 0.01(\mathrm{mg} / \mathrm{L})$ & 200 & 70.9 & 106.4 & 2056 & 106.4 & 113.5 & 156.0 & 177.0 & 142.0 & 1262.1 \\
\hline Dureza total $\pm 0.01\left(\mathrm{mg} / \mathrm{L} \mathrm{CaCO}_{3}\right)$ & 100 & 24 & 60 & 480 & 10 & 224 & 196 & 56 & 89 & 1672 \\
\hline Sólidos sedimentables $\pm 0.01(\mathrm{~mL} / \mathrm{L})$ & 1.0 & 0.1 & 0.5 & 0.1 & 1.0 & 0.1 & 0.33 & 1.0 & 0.1 & 0.1 \\
\hline Coliformes totales $\pm 0.1(\mathrm{NMP} / 100 \mathrm{~mL})$ & 2000 & $>16000$ & $>16000$ & 920 & 3000 & $>1600$ & $<1.8$ & 5000 & $>1600$ & 240 \\
\hline Coliformes fecales $\pm 0.1(\mathrm{NMP} / 100 \mathrm{~mL})$ & 1000 & $<1.8$ & 20 & 220 & 2400 & 20 & $<1.8$ & 70 & 140 & 130 \\
\hline Oxígeno disuelto $\pm 0.01(\mathrm{mg} / \mathrm{L})$ & $>5.0$ & 5.1 & 4.4 & 12.0 & 7.9 & 5.7 & 2.9 & 4.7 & 4.3 & 4.8 \\
\hline \multicolumn{11}{|l|}{ Demanda bioquímica de oxígeno } \\
\hline $5 \pm 0.01(\mathrm{mg} / \mathrm{L})$ & $<20$ & 1000 & 1400 & 104 & 10.0 & 5.44 & 99.9 & 5.1 & 4.3 & 5.0 \\
\hline Nitrato $\pm 0.01(\mathrm{mg} / \mathrm{L})$ & $<5.0$ & 0.5 & 0.5 & 59.2 & 0.5 & $<0.5$ & $<0.5$ & 1.5 & 0.7 & 11.8 \\
\hline Nitrito $\pm 0.01(\mathrm{mg} / \mathrm{L})$ & $<0.05$ & 0.01 & 0.01 & 0.17 & 0.02 & 0.01 & 0.16 & 0.02 & 0.01 & 0.38 \\
\hline Fosfato $\pm 0.1(\mathrm{mg} / \mathrm{L})$ & $<1.0$ & 1.1 & 6.7 & 6.4 & 0.9 & 1.4 & 11.9 & 1.1 & 0.9 & 0.3 \\
\hline
\end{tabular}

$204 \pm 33 \mathrm{mg} / \mathrm{L}$ en ambos monitoreos. Respecto a la conductividad y dureza, el estero presentó aguas de tipo dulce y muy dura, con valores de 978 a $1331 \mu \mathrm{S} /$ $\mathrm{cm}$ en el monitoreo de 2013, mientras que en 2015 paso a ser subsalina y muy dura en E10 $(1520 \mu \mathrm{S} /$ $\mathrm{cm})$, e hiposalina $(7071 \mu \mathrm{S} / \mathrm{cm})$ en su desembocadura en la zona costera (E11), con altos valores de cloruro (213-709 en 2013 y 156-2822 mg/L en 2015. Sin embargo, estos valores pueden estar asociados a los reiterados episodios de marejadas que afectaron a la región en el periodo analizado (UV 2016).

El contenido de coliformes en el estero El Sauce (Fig. 3a1) registró niveles de contaminación fecal significativos en su origen (16 000 NMP en $100 \mathrm{~mL}$ ) (16 000 veces por sobre la norma). Esta situación está relacionada con la contaminación del estero Las Cenizas en F2 y F3 (Fig. 2c, d). En tanto, en su zona media y desembocadura (E8-E11) disminuyó significativamente a valores menores a $33 \mathrm{NMP} / 100$ $\mathrm{mL}$, debido a la acción bactericida del agua de mar (Manahan 2007, Soler et al. 2009). Respecto al contenido de materia orgánica, la figura $\mathbf{3 b 1}$ muestra que en diciembre de 2013 el estero presentó una media de $80.4 \pm 41.1 \mathrm{mg} / \mathrm{L}$ de $\mathrm{DBO}_{5}(\mathrm{p}<0.01)$ en todas las estaciones, siendo la estación E7 la que registró los mayores valores. Éstos disminuyeron notablemente en 2015 en todas las estaciones con excepción de E7 (Fig. 3b2), con una media de $3.2 \pm 0.37 \mathrm{mg} / \mathrm{L}$, bajo los máximos permitidos, mientras que para E7 supera 4.2 veces los LMP. Los resultados mostraron una relación directa con las bajas concentraciones de OD en ambos monitoreos.

Los resultados del contenido de nutrientes de las aguas del estero (Fig. 3c1, d1) presentan altos valores sobre los LMP a lo largo de toda su extensión. Se observa que los compuestos nitrogenados disminuyen a medida que avanza el curso del agua de 12 a $2 \mathrm{mg} / \mathrm{L}$ para nitrato y 0.025 y $2.95 \mathrm{mg} / \mathrm{L}$ para nitrito en diciembre de 2013 y de $1.4 \pm 0.5 \mathrm{mg} / \mathrm{L}$ y 0.11 a $0.38 \mathrm{mg} / \mathrm{L}$ en enero de 2015, no así los fosfatos, con concentraciones altas en la zona media desde $3.58 \mathrm{mg} / \mathrm{L}$ hasta $29.12 \mathrm{mg} / \mathrm{L}$ en E9 en diciembre de 2013. En tanto, en enero de 2015 se registraron las menores concentraciones a lo largo del estero hasta la desembocadura. Estos resultados refuerzan la pérdida de la calidad oligotrófica del estero en toda su extensión, que presenta condiciones eutróficas en su origen y no se logra depurar a medida que avanza en la cuenca debido al ingreso de las aguas de la PTAS, a los aportes difusos de aguas domésticas en la localidad de Laguna Verde y a lixiviados del relleno sanitario Los Molles de la ciudad de Valparaíso.

Smith (2003) y Chalar (2007) plantean que los altos niveles de materia orgánica y nutrientes $(\mathrm{N}-$ $\mathrm{NO}_{3}{ }^{-}$y el $\mathrm{PO}_{4}{ }^{-3}$ ) constituyen la causa principal de eutrofización de un cuerpo de agua (Espósito et al. 

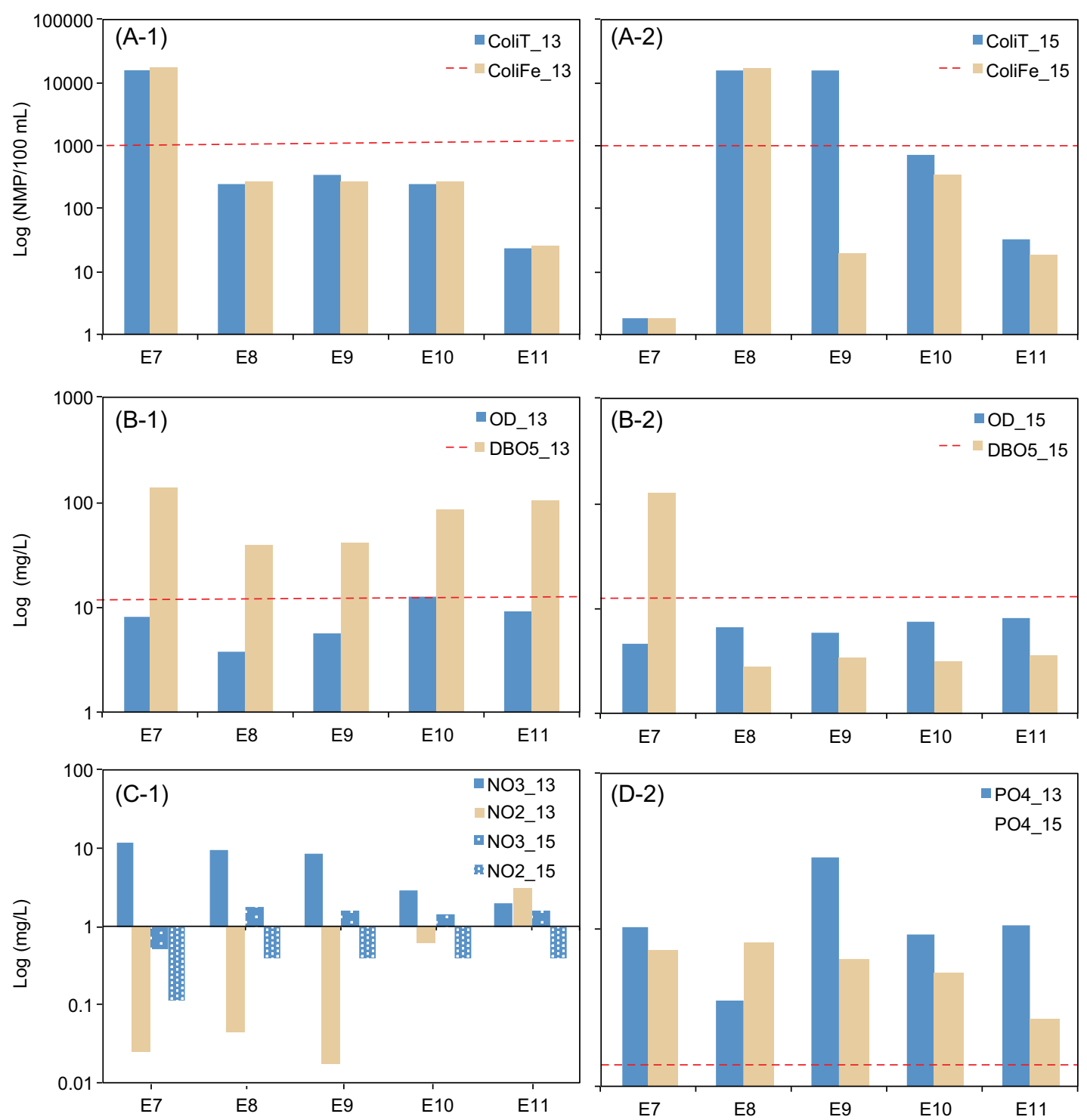

Fig. 3. Resultados de los monitoreos de diciembre de 2013 y enero de 2015 de: (a1, a2) coliformes totales y fecales de; (b1, b2) oxígeno disuelto (OD) y demanda bioquímica de oxígeno $5\left(\mathrm{DBO}_{5}\right)$; (c1) nitrato $\left(\mathrm{NO}_{3}{ }^{-}\right)$y nitrito $\left(\mathrm{NO}_{2}^{-}\right)$; (d1) fosfatos $\left(\mathrm{PO}_{4}^{-3}\right)$

2016), lo cual se acentúa por la disminución de las corrientes e incremento de la profundidad. En el estero El Sauce se registró durante todo el año un crecimiento masivo de microalgas y macrófitas, principalmente en la época estival. El fósforo es un elemento crítico en un cuerpo de agua; en los periodos secos, el mayor aporte de este mineral provendría del flujo de aguas subterráneas y en menor medida de la descarga continua de cursos superficiales (Allison et al. 2014). Por otra parte, en un periodo de mayor escorrentía superficial, los nutrientes serían incorporados a través de la carga de sedimentos producto de la erosión de los suelos, y por los fertilizantes y plaguicidas, los que se transportan en suspensión hacia los cursos de agua (Smith 2003, Abarca 2007).

El ACP muestra una alta variabilidad en las condiciones ambientales, principalmente en el $\mathrm{pH}$ y el contenido de nutrientes (Fig. 4). El análisis explicó el $67.3 \%$ de la varianza para diciembre de 2013 y $51.9 \%$ para enero de 2015. En el primer año, las aguas del estero El Sauce presentaron una alta asociación entre el contenido de SS, color, $\mathrm{pH}$ y coliformes fecales. Por su parte, las fuentes aportantes fueron diferentes entre sí: F6 presentó condiciones de alta conductividad 
eléctrica (CE), contenido de cloruros $\left(\mathrm{Cl}^{-}\right)$, dureza y nitratos, parámetros característicos de vertidos de percolados de vertederos, que por lixiviación habrían contaminado las napas subterráneas. Este aporte se focalizó en la zona baja de la cuenca, donde se ubica el relleno sanitario Los Molles, generando un impacto directo en las estaciones E10 y E11. Las estaciones
F3 y F2 estarían directamente influenciadas por el vertido de las aguas servidas tratadas en la empresa sanitaria (Fig. 4). Estas aguas se caracterizaron por un alto contenido de materia orgánica y altas concentraciones de coliformes, los cuales modificaron la calidad del agua en las estaciones E7, E8 y E9. En enero de 2015, la estación F6 se identificó como
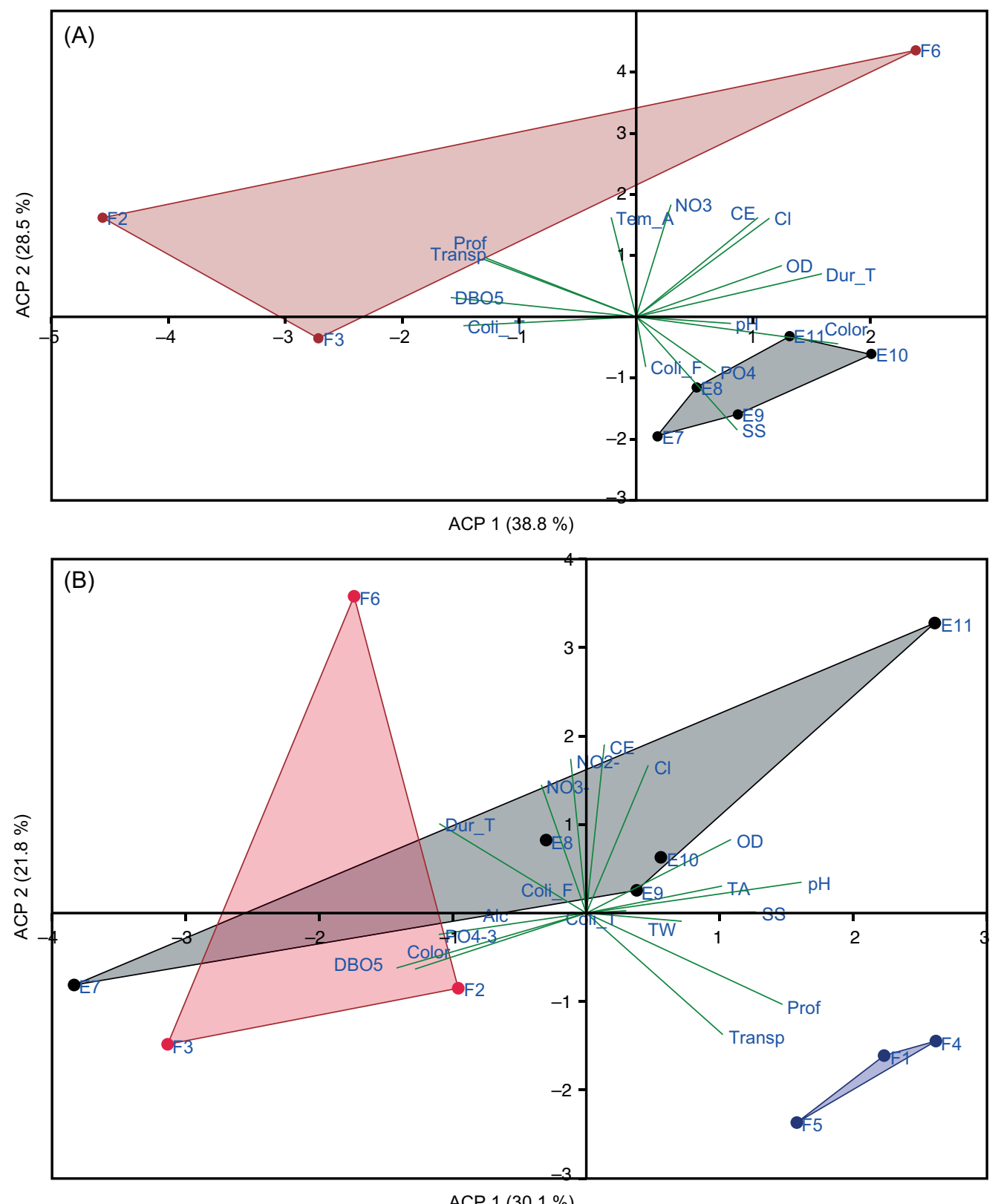

Fig. 4. Análisis de componentes principales (ACP) de las variables ambientales para ambos monitoreos. (a) 2013, (b) 2015. Rosa (•): fuentes industriales aportantes; gris (•): estaciones del estero El Sauce; azul $(\bullet)$ : humedales 
aportadora de iones con altos valores de $\mathrm{CE}, \mathrm{Cl}^{-} \mathrm{y}$ nutrientes nitrogenados, en tanto que F2 y F3 mostraron altos niveles de materia orgánica, color y fosfatos. Destacan las estaciones F1, F4 y F5 con aguas muy diferentes a las del estero.

\section{CONCLUSIONES}

El estero El Sauce es un cuerpo de agua somero e hipertrófico, y sus aguas, en casi toda su extensión, corresponden a la categoría de mala calidad, ya que presentan un alto contenido de materia orgánica, nutrientes, cloruros y contaminación fecal. Por este motivo, las aguas de su cuenca no cumplen con la normativa ambiental para ningún uso. La causa principal de este deterioro sería el ingreso no puntual de aguas servidas en el sector de Placilla y la descarga puntual de las aguas de la planta de tratamiento de aguas servidas ESVAL, que aumentó su caudal en aproximadamente $70 \%$, con aguas altamente contaminadas que no cumplen con la norma de emisión.

Una segunda causa sería el ingreso en su parte media de líquidos percolados desde un antiguo vertedero y actual relleno sanitario de la región de Valparaíso. Estas aguas no sólo contaminan el estero sino también las napas subterráneas del acuífero en la localidad de Laguna Verde, del cual se abastece sin tratamiento más del $60 \%$ de dicha comunidad.

Una tercera causa sería que las viviendas de la localidad rural no poseen red de alcantarillado y contaminan el estero como fuentes no puntuales, principalmente en la zona cercana a su desembocadura.

Las principales causas del alto grado de deterioro que exhibe la cuenca del estero El Sauce podría revertirse mediante la fiscalización de las autoridades competentes y el cumplimiento de las normas de emisión para aguas continentales DS 90 (MSGP-SEGPRES 2000). Esto, además, de un plan de mitigación de las aguas que percolan desde el relleno sanitario Los Molles y la implementación de alcantarillado en la población rural ribereña, dado el alto riesgo ambiental y de salud que implica el uso actual de las aguas de este importante y escaso recurso.

\section{AGRADECIMIENTOS}

Se agradece el apoyo recibido a través de los proyectos DGI Regular CNEI 04-15/16, Dirección de Investigación y Programa de Biodiversidad de la Facultad de Ciencias Naturales y Exactas de la Universidad de Playa Ancha.

\section{REFERENCIAS}

Abarca F.J. (2007). Técnicas para evaluación y monitoreo del estado de los humedales y otros ecosistemas acuáticos. En: Perspectivas sobre la conservación de ecosistemas acuáticos en México (Sánchez O., Herzig M., Peters E., Márquez R. y Zambrano L., Eds.). Instituto Nacional de Ecología, Ciudad de México, México, pp. 113-144.

Allison O., Randy D. y Michael D. 2014. The upsidedown river: Reservoirs, algal blooms, and tributaries affect temporal and spatial patterns in nitrogen and phosphorus in the Klamath River, USA. J. Hydro. 519 (Part A), 164-176.

DOI: 10.1016/j.jhydrol.2014.06.025

APHA (2005). Standard methods for examination of water and wastewater. 21a ed. American Public Health Association/American Water Work Association/Water Pollution Control Federation. Washington D.C., EUA, $1325 \mathrm{pp}$.

Aranda C. (2013). Plan maestro de recualificación sostenible en Laguna Verde. Tesis de Licenciatura. Facultad de Arquitectura y Urbanismo, Universidad de Chile. Santiago, Chile, 50 pp.

Azpilcueta M., Pedroza A., Sánchez I., Salcedo M. y Trejo R. (2017). Calidad química del agua en un área agrícola de maíz forrajero (Zea mays L.) en la Comarca Lagunera, México. Rev. Int. Contam. Ambie. 33 (1), 75-83. DOI: 10.20937/RICA.2017.33.01.07

Cataldo A.M. (2015). Evaluación de la calidad de agua del estero Las Cenizas mediante parámetros físicos, químicos y microbiológicos. Tesis de Licenciatura. Facultad de Ciencias Naturales y Exactas, Universidad de Playa Ancha. Valparaíso, Chile, 131 pp.

Chalar G. (2007). Dinámica de la eutrofización a diferentes escalas temporales: Embalse Salto Grande (Argentina-Uruguay). En: Eutrofización en América del Sur: causas, consecuencias y tecnología de gerenciamiento y control (Galicia J., Matsumura T. y Sidagis C., Eds.). Instituto Internacional de Ecología y Gerenciamiento Ambiental, San Carlos, Brasil, pp. 87-101.

Chapman D. y Kimtsach V. (1996). Selection of water quality variables. Chapter 3 . En: Water quality assessments - A guide to use of biota, sediments and water in environmental monitoring. (D. Chapman, Ed.). 2a ed. UNESCO/WHO/UNEP, Londres, Inglaterra, pp. 74-133.

DOI: 10.4324/NOE0419216001

CONAMA (2004). Guía para el desarrollo de normas para la protección de las aguas continentales superficiales. Comisión Nacional del Medio Ambiente. Santiago, Chile, 18 pp. 
De Groot R., Stuip M., Finlayson M. y Davidson N. (2007). Valoración de humedales, lineamientos para valorar los beneficios derivados de los servicios de los ecosistemas de humedales. Informe Técnico de Ramsar $\mathrm{N}^{\circ} 3$ y N ${ }^{\circ}$ 27. Cuaderno técnico, convenio sobre la diversidad biológica. Secretaría de la Convención de Ramsar, Gland, Suiza, 58 pp.

De la Lanza G. (2000). Criterios generales para la elección de bioindicadores. En: Organismos indicadores de la calidad del agua y de la contaminación (biondicadores) (de la Lanza G., Hernández S. y Carvajal J. L., Eds.). Ciudad de México, México, pp. 17-42.

De la Lanza G. (2002). Recursos hidrológicos de México. En: Manual para el manejo y conservación de los humedales en México (Abarca J. y Herzig M., Eds.). 3a. ed. Fínix, Estados Unidos, pp. 113-144.

DGA (2005). Informe técnico evaluación de los recursos subterráneos de las cuencas costeras de la Quinta Región. Informe de actualización. Serie de Documentos Técnicos S.D.T. No 201. Dirección General de Aguas, Ministerio de Obras Públicas. Santiago, Chile, 93 pp.

DGA (2016). Información pluviométrica, fluviométrica, estado de embalses y aguas subterráneas. Boletín $\mathrm{N}^{\circ}$ 461, Sistema de Seguimiento Documentos S.S.D. N ${ }^{\circ}$ 10279238. Dirección General de Aguas, Ministerio de Obras Públicas. Santiago, Chile, 32 pp.

Espósito M.E., Blanco M., Sequeira M.E., Paoloni J.D. Fernández S.N., Amiotti N.M. y Díaz S.L. (2016). Contaminación natural (As, F) y eutrofización (N, P) en la cuenca del arroyo El Divisorio, Argentina. Phyton 85 (1), 51-62.

Figueroa R., Palma A., Ruíz V. y Niell X. (2007). Análisis comparativo de índices bióticos utilizados en la evaluación de la calidad de las aguas en un río mediterráneo de Chile: Río Chillán, VIII Región. Rev. Chil. Hist Nat. 80 (2), 225-242.

DOI: $10.4067 / \mathrm{S} 0716-078 X 2007000200008$

Figueroa R., Suárez M.L., Andreu A., Ruiz V.H. y VidalAbarca M.R. (2009). Caracterización ecológica de humedales de la zona semiárida en Chile central. Gayana 73, (1), 76-94.

DOI: $10.4067 / \mathrm{S} 0717-65382009000100011$

Hammer Ø., Harper D.A. y Ryan P.D. (2001). PAST: Paleontological statistics software package for education and data analysis. Paleontología Electrónica 4 (1), 1-9 pp.

Hargrave B.T., Holmer M. y Newcombe C.P. (2008). Towards a classification of organic enrichment in marine sediments based on biogeochemical indicators. Mar. Pollut. Bull. 56 (5), 810-824.

DOI: 10.1016/j.marpolbul.2008.02.006

Inostroza C.I. y Ruiz C.M. (2015). Caracterización y evaluación de la calidad del agua de pozo en Laguna
Verde, región de Valparaíso. Tesis de Licenciatura. Facultad de Ciencias Naturales y Exactas, Universidad de Playa Ancha. Valparaíso, Chile, 137 pp.

Jongman R., Ter Braak C. y van Tongeren O. (1987). Data analysis in community and landscape ecology. Cambridge University Press, Cambridge, Inglaterra, 299 pp.

León H., Cruz C., Dávila R., Velasco F. y Chapa J. (2015). Impacto del lixiviado generado en el relleno sanitario municipal de Linares (Nuevo León) sobre la calidad del agua superficial y subterránea. Rev. Mex. Cienc. Geol. 3 (32), 514-526.

Luna V., Alva A. y Bernal A. (2004). Cuerpos de agua superficiales. En: Técnicas de muestreo para manejadores de recursos naturales (Bautista F., Ed.). Universidad Nacional Autónoma de México, Dirección General de Estudios de Posgrado/Universidad Autónoma de Yucatán/Consejo Nacional de Ciencia y Tecnología e Instituto Nacional de Ecología, México, pp. 117-143.

Manahan S.E. (2007). Introducción a la química ambiental. Editorial Reverté. México, D.F. México, 725 pp.

Millanir N. (2003). Proyecto de factibilidad de instalación de redes de alcantarillado y agua potable sector Curaumilla-Laguna Verde. Tesis de Licenciatura. Departamento de Diseño y Manufactura, Universidad Técnica Federico Santa María. Viña del Mar, Chile, $90 \mathrm{pp}$.

Moreira-Muñoz A. y Salazar A. (2014). Reserva de la Biosfera La Campana-Peñuelas: micro-región modelo para la planificación del desarrollo regional sustentable. En: Reservas de la biosfera de Chile: Laboratorios para la sustentabilidad (Moreira-Muñoz A. y Borsdorf A., Eds.). Instituto de Geografía, Pontificia Universidad Católica de Chile, Instituto Interdisciplinario de Investigación sobre la montaña, Academia de Ciencias Austriacas. Santiago, Chile, pp. 106-122.

Montes C. y Martino P. (1987). Las lagunas salinas españolas. En: Bases científicas para la protección de humedales en España (Real Academia de Ciencia Exactas, Físicas y Naturales, Ed.). Madrid, España, pp. 95-145.

MOP (1978). Norma Chilena Oficial 1333/78. Requisitos de calidad del agua para diferentes usos. Instituto Nacional de Normalización por Decreto Supremo $\mathrm{N}^{\circ}$ $867 / 78$ y modificada por Decreto $N^{o} 105 / 78$. Ministerio de Obras Públicas. Publicado en el Diario Oficial $\mathrm{N}^{\circ}$ 30.107 del 5 de julio de 1978 y modificación del 22 de mayo de 1987.

MOP (1996). Norma Chilena Oficial 411/2. Calidad de agua - muestreo - parte 2: Guía sobre técnicas de muestreo. Norma Chilena Oficial de la República por Decreto Supremo No 501/78. Ministerio de Obras Públicas, publicado en el Diario Oficial № 35.532. 2 de agosto. 
MSGP-SEGPRES (2000). Decreto Supremo No 90. Norma de emisión para la regulación de contaminantes asociados a las descargas de residuos líquidos a aguas marinas y continentales superficiales. Ministerio Secretaría General de la Presidencia, Santiago, Chile. Diario Oficial del 7 de marzo.

Navarro C. (2013). Memorial de territorio: las aguas que nos hablan del origen. Tesis de Licenciatura. Escuela de Arquitectura y Diseño Pontificia, Universidad Católica de Valparaíso. Valparaíso, Chile, 225 pp.

OMS (2005). Guías para la calidad del agua potable. Recomendaciones. Primer apéndice a la 3a edición, vol. 1. Organización Mundial de la Salud. Ginebra, Suiza, 408 pp.

Pavez J., Silva N., Cornejo-D’Ottone M. y Rivera C. (2018). Laguna Verde Bay's sediments origin and its heavy metals content ( $\mathrm{Al}, \mathrm{Fe}, \mathrm{Cu}, \mathrm{Mn}, \mathrm{Pb}$ and $\mathrm{Hg}$ ), Valparaíso, Chile. Lat. Am. J. Aquat. Res. 46 (5), 11161122. DOI: $10.3856 /$ vol46-issue5-fulltext-24

PLADECO (2006). Diagnóstico comunal Valparaíso. Ilustre municipalidad de Valparaíso. Plan de desarrollo comunal de Valparaíso. Documento oficial, Valparaíso, Chile, 508 pp.

Silob (2012). ITE. AES/02/2012. Caracterización de aguas, Sector Laguna Verde, Quinta Región. Silob Chile. Informe técnico preparado por el Departamento de Ingeniería Ambiental, Valparaíso, Chile, 18 pp.

SISS (2013). Resultados fiscalización de plantas de tratamiento de aguas servidas, Chile. Año 2013. Superintendencia de Servicios Sanitarios. [en línea]. http:// www.siss.gob.cl/586/w3-propertyvalue-6408.html. 25/05/2014.

Smith V.H., Tilman D. y Nekola J.C. (1999). Eutrophication: Impact on excess nutrient inputs on freshwater, marine and terrestrial ecosystems. Environ. Pollut. 100 (1-3), 179-196. DOI: 10.1016/S0269-7491(99)00091-3

Smith V.H. (2003). Eutrophication of freshwater and coastal marine ecosystems. A global problem. Environ. Sci. Pollut. R. 10 (2), 126-139.

DOI: $10.1065 /$ espr2002.12.142
Soler M., Lobos S., Lorca M. y Navarrete E. (2009). Enterovirus en aguas naturales de Valparaíso: una propuesta metodológica para su análisis. Rev. Biol. Mar. Oceanog. 44 (2), 511-516. DOI: $10.4067 / \mathrm{S} 0718-19572009000200023$

Schindler D.W. (2006). Recent advances in the understanding and management of eutrophication. Limnol. Oceanogr. (51), 356-363. DOI: $10.4319 /$ lo.2006.51.1_part_2.0356

Tobar T. y Torres C.L. (2014). Evaluación de la calidad del agua del estero El Sauce, Laguna Verde: Impacto y Consecuencias. Tesis de Licenciatura. Facultad de Ciencias Naturales y Exactas, Universidad de Playa Ancha. Valparaíso, Chile, 175 pp.

Tóth J. (2000). Las aguas subterráneas como agente geológico: causa, procesos y manifestaciones. Boletín Geológico y Minero 111 (4), 9-26.

UNESCO (2017). Informe mundial de las Naciones Unidas sobre el desarrollo de los recursos hídricos. Aguas residuales: el recurso desaprovechado. Organización de las Naciones Unidas para la Educación, la Ciencia y la Cultura. París, Francia, 202 pp.

UV (2016). Atlas de oleaje de Chile. Universidad de Valparaíso [en línea]. http://www.oleaje.uv.cl/19/12/2016

Wolanski E., Boorman L.A., Chícharo L., Langlois-Saliou E., Lara R., Plater A.J., Uncles R. J. y Zalewski M. (2004). Ecohydrology as new tool for sustainable management of estuaries and coastal waters. Wetl. Ecol. Manag. 12 (4), 235-276. DOI: $10.1007 / \mathrm{s} 11273-005-4752-4$

Zunino S., Aliaga C. y Da Venezia P. (2009). Comunidades de peces en desembocaduras de ríos y esteros de la región de Valparaíso, Chile central. Rev. Biol. Mar. Oceanog. 44 (1), 123-130.

DOI: $10.4067 / \mathrm{S} 0718-19572009000100011$

Zúñiga M. (2015). Caracterización de la vulnerabilidad socio territorial en el área rural, forestal y de expansión urbana de Laguna Verde, Valparaíso. Tesis de Licenciatura. Departamento de Geografía, Facultad de Ciencias Naturales y Exactas, Universidad de Playa Ancha, Valparaíso, Chile, 162 pp. 